\title{
ARTICLE
}

\section{Automatic quantitative analysis of experimental primary and secondary retinal neurodegeneration: implications for optic neuropathies}

\author{
BM Davis ${ }^{1}, \mathrm{~L} \mathrm{Guo}^{1}$, J Brenton ${ }^{1}$, L Langley ${ }^{1}, \mathrm{EM}$ Normando ${ }^{2}$ and MF Cordeiro ${ }^{1,2}$
}

\begin{abstract}
Secondary neurodegeneration is thought to play an important role in the pathology of neurodegenerative disease, which potential therapies may target. However, the quantitative assessment of the degree of secondary neurodegeneration is difficult. The present study describes a novel algorithm from which estimates of primary and secondary degeneration are computed using wellestablished rodent models of partial optic nerve transection (pONT) and ocular hypertension (OHT). Brn3-labelled retinal ganglion cells (RGCs) were identified in whole-retinal mounts from which RGC density, nearest neighbour distances and regularity indices were determined. The spatial distribution and rate of RGC loss were assessed and the percentage of primary and secondary degeneration in each non-overlapping segment was calculated. Mean RGC number (82 592 \pm 681 ) and RGC density (1695 \pm 23.3 $\mathrm{RGC} / \mathrm{mm}^{2}$ ) in naïve eyes were comparable with previous studies, with an average decline in RGC density of $71 \pm 17$ and $23 \pm 5 \%$ over the time course of pONT and OHT models, respectively. Spatial analysis revealed greatest RGC loss in the superior and central retina in pONT, but significant RGC loss in the inferior retina from 3 days post model induction. In comparison, there was no significant difference between superior and inferior retina after OHT induction, and RGC loss occurred mainly along the superior/ inferior axis ( 30\%) versus the nasal-temporal axis ( 15\%). Intriguingly, a significant loss of RGCs was also observed in contralateral eyes in experimental OHT. In conclusion, a novel algorithm to automatically segment Brn3a-labelled retinal whole-mounts into nonoverlapping segments is described, which enables automated spatial and temporal segmentation of RGCs, revealing heterogeneity in the spatial distribution of primary and secondary degenerative processes. This method provides an attractive means to rapidly determine the efficacy of neuroprotective therapies with implications for any neurodegenerative disorder affecting the retina.
\end{abstract}

Cell Death Discovery (2016) 2, 16031; doi:10.1038/cddiscovery.2016.31; published online 23 May 2016

\section{INTRODUCTION}

Secondary neurodegeneration is reported to play a significant role in many diseases of growing socioeconomic and health-economic concern, including neurodegenerative diseases, spinal cord injuries and optic neuropathies. ${ }^{1-7}$ It is thought to occur through a combination of excitotoxicity, inflammation and oxidative stress pathways, which provide attractive therapeutic targets as secondary neurodegeneration is clinically more modifiable than primary. ${ }^{8-10}$ Although substantial progress has been made in our understanding of these disorders in recent years, including recognition of similarities between these conditions, ${ }^{11,12}$ there are presently few effective disease-modifying therapies to slow or reverse the course of neurodegeneration.

One of the most common ophthalmic neurodegenerative diseases is glaucoma, which comprises a distinctive group of progressive optic neuropathies and is the leading cause of irreversible blindness globally. ${ }^{13}$ Gradual degeneration of RGCs and optic nerve damage, with characteristic changes in appearance of the optic disc, is responsible for the progressive visual field loss. Classically (but not exclusively), this manifests as an initial permanent loss of peripheral vision, leading to central vision defects as the disease progresses. ${ }^{14,15}$ Presently, there is no curative treatment for glaucoma, with existing therapies targeting raised intraocular pressure (IOP), the major and modifiable risk factor. ${ }^{16,17}$ Glaucoma is a complex and multifactorial disease, with variation in disease progression, and vision loss continuing to occur in some patients, despite well-controlled IOPs, ${ }^{18}$ suggesting that secondary RGC degeneration plays an important role in glaucoma pathology. ${ }^{10,19}$

Rodent models of glaucoma have proven essential in understanding disease progression and assessing efficacy of therapeutic interventions. In this respect, quantitative assessment of RGC loss is a commonly used end point to assess experimental glaucomatous degeneration and therapeutic intervention efficacy. ${ }^{10,20}$ Although none are exact models of human disease, they allow study of different mechanistic aspects. ${ }^{21}$ Partial optic nerve transection (pONT) is a useful model first described in cynomolgus monkeys and Wistar rats. ${ }^{7,22}$ This model facilitates segregation of primary and secondary degeneration processes by transecting only the superior optic nerve, enabling mechanisms of RGC degeneration and efficacy of therapeutic interventions to be assessed. ${ }^{10,23,24}$ Another, well-established rodent model of glaucoma is the Morrison model of ocular hypertension (OHT), which involves injection of hypertonic saline into the episcleral veins to induce elevated IOP via induction of sclerosis of the trabecular meshwork impeding aqueous drainage. ${ }^{25}$ The resulting elevated 


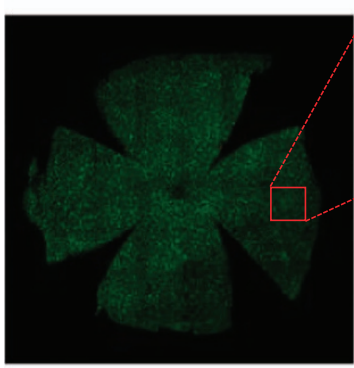

RGC segmentation b

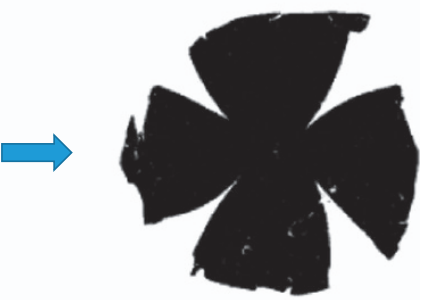

Retinal area segmentation

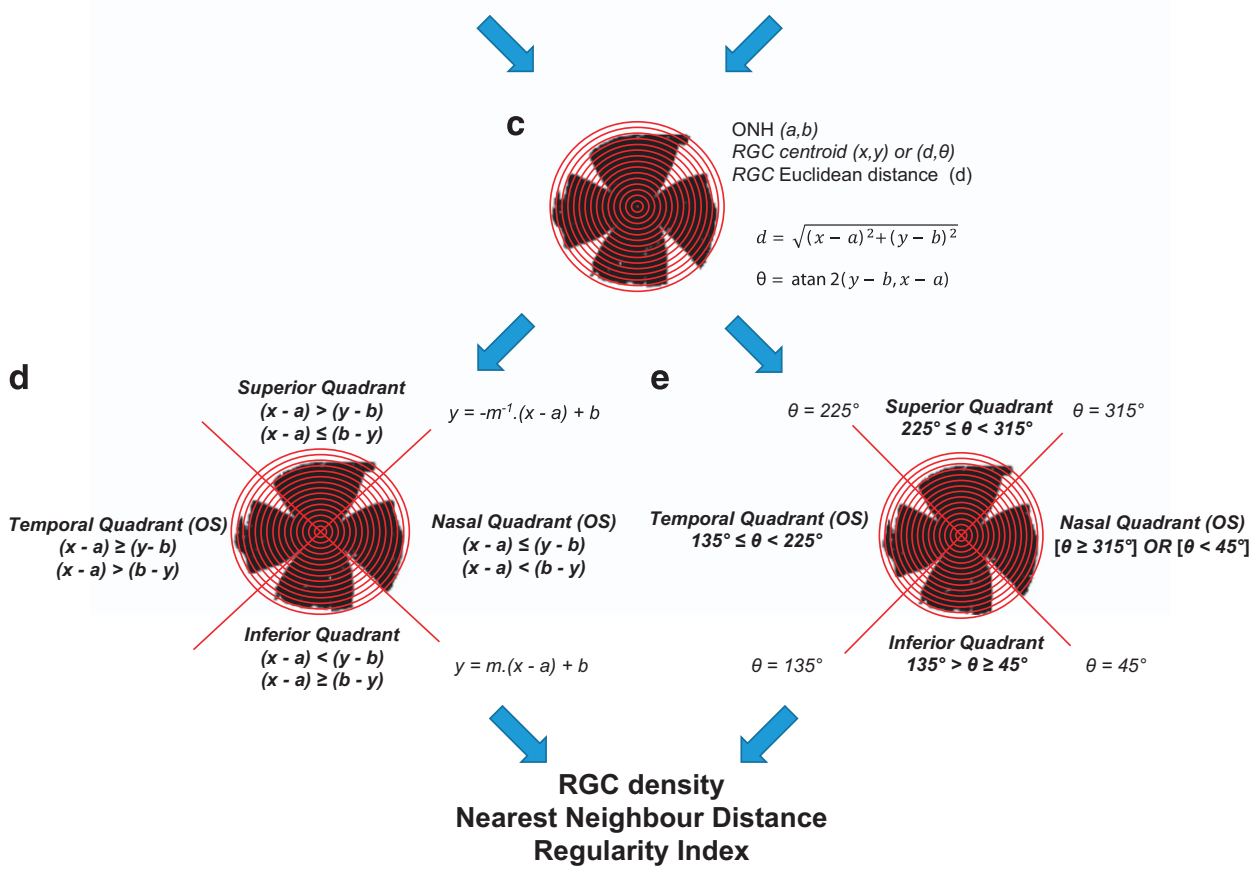

Figure 1. Diagram of RGC segmentation and counting used in this study. (a) Identification of RGC number, area and centroid from Brn3alabelled retinal whole-mounts was completed using previously described methods. ${ }^{10}$ (b) Retinal area was determined by first creating a binary mask of the whole-mount area before applying a customised ImageJ script to measure the retinal area (black pixels) in each retinal segment (ring and quadrants). (c) On defining the centre of the $\mathrm{ONH}$, a series of 15 concentric rings with $0.3 \mathrm{~mm}$ radii were drawn and the position of each RGC determined by calculating the Euclidean distance $[D]$ of each RGC relative to the ONH using ring boundaries as thresholds. RGCs were further segmented into quadrants (d and e) (where $m=b / a$ ) defined using Cartesian (d) or polar coordinates (e) which gave identical results. For each segment, in each case RGC density, average nearest neighbour distance and regularity index were calculated as described in the text.

IOP is correlated with increased RGC apoptosis ${ }^{26,27}$ and functional assessment of RGC loss suggests moderate sustained elevation of IOP results in peripheral vision loss. ${ }^{28}$

Brn3a is a POU-domain transcription factor considered a good histological RGC marker, due to its high RGC specificity and good agreement with retrograde axonal tracer Fluoro-Gold $(97 \%$ RGC co-labelling in rats). ${ }^{29}$ As Brn3a is localised to RGC nuclei, immunohistochemistry of retinal whole-mounts yields images from which RGCs can be readily segmented (Figure 1a). Quantification of Brn3a RGCs is frequently obtained by manually counting retinal segments, ${ }^{7,30-33}$ yet a limitation of this approach is the high variability in RGC density between central and peripheral rodent retina, which can complicate RGC quantification by sampling. ${ }^{34}$ To address these concerns, whole retinas need to be assessed and several groups, including our own, have developed tools to automatically count Brn3a-labelled RGCs, results of which are most frequently presented quantitatively as average whole-mount RGC density $\left(\mathrm{RGC} / \mathrm{mm}^{2}\right)$ or qualitatively as isodensity maps. ${ }^{10,29,35,36}$
In this study we describe a new segmentation algorithm for automatic determination of regional RGC density, nearest neighbour distance (NND) and regularity index (RI), using a series of 15 concentric rings centred on the optic nerve head $(\mathrm{ONH})$, segmented into quadrants. We apply this method to investigate the patterns and natural history of RGC loss in two wellestablished models of glaucoma: the Morrison's model of $\mathrm{OHT}^{25}$ and $\mathrm{pONT}^{23}$ to assess primary and secondary neuronal degenerative processes as potential therapeutic targets.

\section{RESULTS}

Establishment of a novel algorithm for whole-retinal RGC analysis and spatial segmentation

Using the previously established automated RGC counting algorithm, ${ }^{10}$ a method was established to spatially segment Brn3a-labelled retinal whole-mounts. This enabled the spatial and temporal distribution of RGC loss to be automatically determined 
(Figure 1). This script was initially used to evaluate a series of control eyes: average retinal area $(52.4 \pm 5.1 \mu \mathrm{m})$, Brn3a positive RGC number (82 592 \pm 681 RGCs/retina) and average whole-retinal RGC density $\left(1695 \pm 23.3 \mathrm{RGC} / \mathrm{mm}^{2}\right)$ were documented.

Established retinal neuronal mosaic parameters include NNDs and Regularity Indices (RIs). NND refers to the distance between each neuron and its nearest neighbour in the mosaic, presented as the mean NND. ${ }^{37} \mathrm{RI}$ presents one of the most popular methods for quantifying retinal mosaics and comprises a spatial statistic derived from the frequency distribution of NNDs, and is calculated by dividing the mean NND by the standard deviation of the NDD of a population within a sample field (equation (1)). ${ }^{38}$ As orderly retinal mosaics possess an approximate Gaussian NND frequency distribution and random retinal mosaics possess a Poisson distribution, greater RI ratios will be observed for Gaussian (ordered) distributions of retinal cells. ${ }^{39}$ Typically, the RI for neurons arranged randomly is 1.9 , and the more regular the arrangement, the higher the $\mathrm{RI}^{37}$ The RI for retinal mosaics observed to date, is reported to be between 3 and $8 .^{38}$ However, the exact threshold for determining whether the mosaic is non-randomly arranged depends on the number of neurons and the geometry of the field. In addition, the physical size of soma may introduce lower limits (an exclusion radius) onto NNDs which may adversely influence RI calculations. ${ }^{37}$ For control DA rat retina, an average RI of $2.86 \pm 0.03$ was obtained, which is indicative of a retinal mosaic with a regular arrangement. ${ }^{37}$

Application of the whole-retinal algorithm for the spatial and temporal assessment of the RGC mosaic in rodent models of optic neuropathy

To spatially segment retinal RGC populations, the centroid of each Brn3a-labelled RGC was next used to calculate its position relative to the $\mathrm{ONH}$ using vector coordinates, before a series of 15 concentric rings subdivided into four quadrants were constructed from which regional RGC density, NND and RI parameters were determined (Figures 1c-e). This RGC segmentation algorithm was applied to a total of 83 whole-mounted Brn3a-labelled retina 3, 7, 21 and 56 days after pONT induction or 7, 21, 56 and 84 days post OHT induction. A significant reduction in RGC density was observed from 3 days of pONT model induction and after 7 days of OHT model induction, and RGC density declined by $71 \pm 11$ and $23 \pm 5 \%$ over the course of pONT and OHT models, respectively, compared with a population of naïve eyes (Table 1). Upon completion of RGC segmentation, NNDs were computed from each Brn3a positive RGC, and combined with RGC centroid data to produce qualitative NND colour maps (Figure 2a), where the smallest NNDs (red) represent regions of greatest RGC density, larger NNDs (green/blue) regions of low RGC density. Quantitative analysis of normalised NND distributions (Figures $2 \mathrm{~b}-\mathrm{i}$ ) reveals that over the course of pONT and OHT models, overall NND distribution shifted to greater values, suggestive of diffuse RGC loss (primary degeneration). ${ }^{40,41}$ The concurrent increase in skew of the NND distribution to higher NND values (Figures $2 \mathrm{~b}-\mathrm{e}$ ) versus naive controls is evidence of an additional component of clustered RGC loss, which may be indicative of secondary degenerative processes. ${ }^{40,41}$

RGCs loss occurs predominantly in the superior-central retinal in the pONT model

We next analysed the pattern of RGC loss in the pONT model and its progression over time (Figure 3a). A significant loss of RGC density occurs rapidly from 3 days after model induction which plateaus by 56 days $(P<0.001$; Table 1$)$. This effect is mirrored by a delayed but significant increase in RGC NND (Figure 3b) from baseline to 7 days post pONT induction (14.42 \pm 0.08 versus $16.88 \pm 0.75 \mu \mathrm{m}, P<0.05)$. Rl appears initially insensitive to pONT model induction ( 3 days post pONT) but as the model progresses, from 7 days onwards $(2.55 \pm 0.09, P<0.01)$, Rl values begin to decline (Figure $3 \mathrm{c}$ ). By 21 days post pONT induction, RI approaches

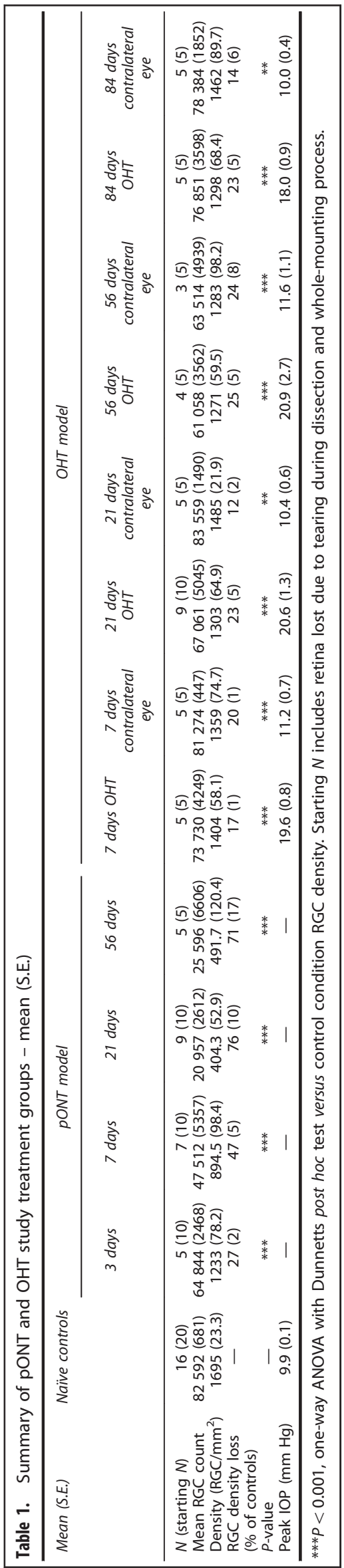



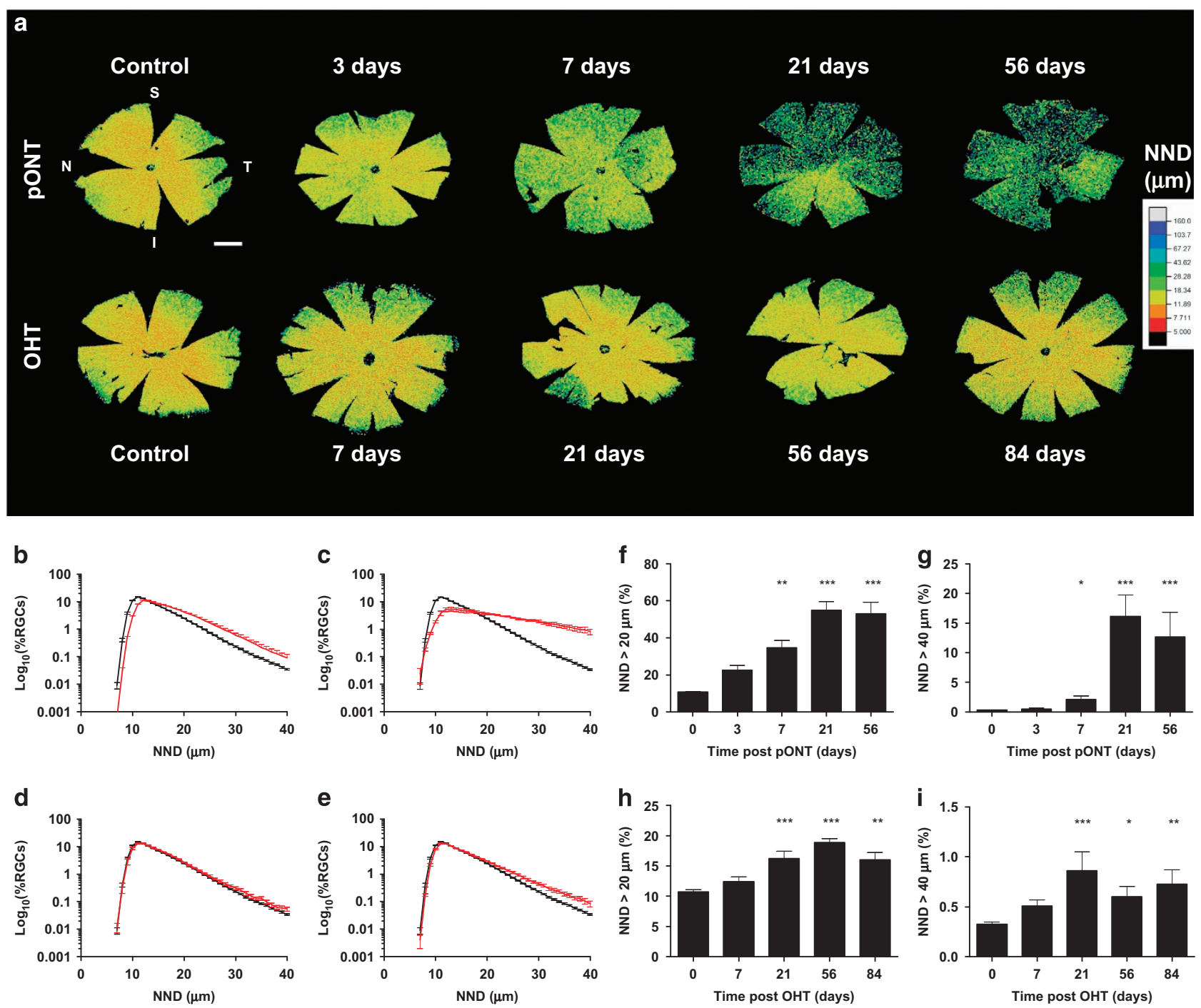

Figure 2. Change in NND distribution over the course of pONT and OHT models. (a) Colour maps comparing changes in RGC nearest neighbour distance in pONT and OHT models over time, scale bar $=2 \mathrm{~mm}$. (b-e) Longitudinal changes in global NND distribution over the course of the pONT (b and $\mathbf{c}$ ) and OHT (d and e) models (red) versus naïve controls (black). (f-i) Graphs illustrating the proportion of the normalised NND distribution with NNDs (b and $\mathbf{c}$ ) greater than $20 \mu \mathrm{m}$ ( $\mathbf{f}$ and $\mathbf{h}$ ) and $40 \mu \mathrm{m}(\mathbf{g}$ and $\mathbf{i})$ in the pONT (f and $\mathbf{g})$ and OHT (h and $\mathbf{i})$ models. Difference versus naïve controls determined using a one-way ANOVA with Dunns post hoc test.

1.9 which is indicative of a retinal mosaic comprising randomly distributed RGCs. ${ }^{37}$ Further segmentation of the pONT retinal wholemounts into quadrants (Figures $3 \mathrm{~d}-\mathrm{f}$ ) reveals that significantly greater loss of RGC density was observed in the superior quadrant (Figure 3d, $P<0.001)$ at 21 and 56 days post model induction. This pattern of RGC loss was reflected in significant differences in NND (Figure 3e, $P<0.01$ ) and Rl values (Figure 3f, $P<0.01$ and $P<0.05$ for 21 and 56 days, respectively). Further segmentation of superior and inferior retinal quadrants into 15 concentric rings suggests that RGC loss in both superior and inferior sectors progresses quickly after pONT model induction and is primarily concentrated in the central superior retina.

RGC loss predominantly occurs along the superior-inferior axis in the OHT model

The decline in RGC density observed in the Morrison's OHT model was less than that observed in the pONT model (Figure $4 a$ and Table 1). IOP peaked $24 \mathrm{~h}$ post $\mathrm{OHT}$ model induction with an average IOP of $19.9 \pm 3.2$ versus $12.0 \pm 4.0 \mathrm{~mm} \mathrm{Hg}$ in untreated contralateral eyes. A significant increase in IOP was observed in all
OHT eyes $(P<0.05)$ versus untreated contralateral eyes, similar to those previously reported by our group. ${ }^{42} \mathrm{~A}$ significant loss of RGC density was observed from 7 days post OHT induction (Figures 4a, $P<0.001$ ), which was accompanied by significant increases in NND (Figure $4 \mathrm{~b}$ ) from 21 days (14.42 \pm 0.08 versus $15.71 \pm 0.28 \mu \mathrm{m}$ for control and 21 days post OHT model induction). RI exhibited only a mild decline over the course of this model (Figure 4c), only achieving significance at 84 days post OHT induction (2.86 \pm 0.03 versus $2.55 \pm 0.11, P<0.05$ for controls and 84 days post $\mathrm{OHT}$ model induction, respectively), suggesting retinal mosaic regularity was largely preserved, likely due to the relatively mild IOP elevation in this model.

Segmentation of the OHT retinal whole-mounts into quadrants (Figures $4 d-f$ ) reveals that RGC loss occurs at a similar rate throughout the entire retina, with no significant difference in RGC density (Figure 4d), NND (Figure 4e) or RI (Figure 4f) in superior and inferior quadrants at any time point investigated. Further segmentation of superior and inferior quadrants into 15 concentric rings reveals that RGC density declines more rapidly in the retinal centre than in the periphery (Figure $4 \mathrm{~g}$ ). This observation is mirrored by more pronounced increases in NND and declines in RI 

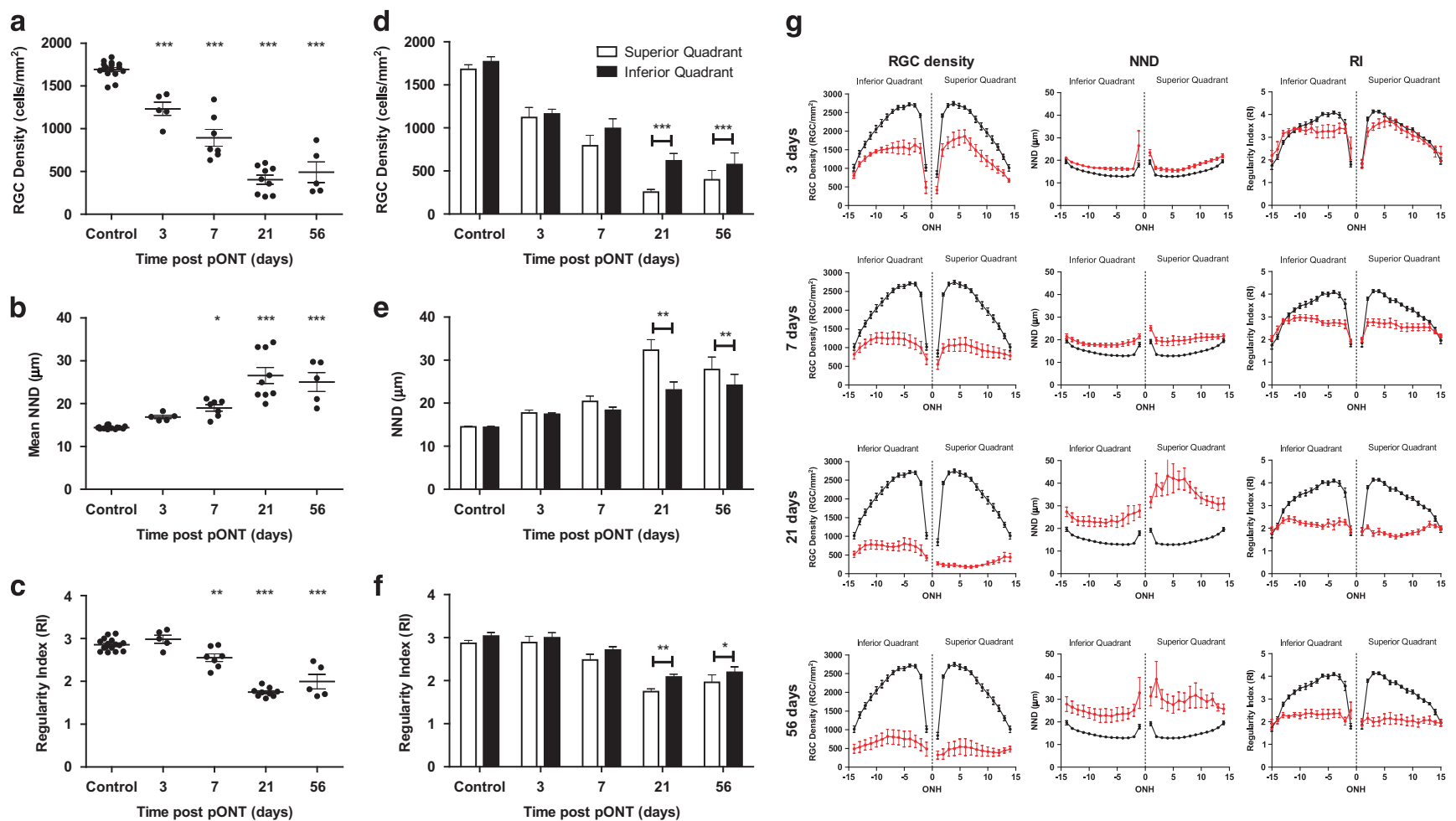

Figure 3. Retinal ganglion cell (RGC) survival after partial optic nerve transection (pONT) in the DA rat. Whole-retinal profiles of pONT induced loss of (a) RGC density, (b) nearest neighbour distance and (c) regularity index demonstrating significant reducing in RGC populations and retinal mosaic regularity over the course of this model (one-way ANOVA with Dunnets' post hoc test versus naïve controls). (d-f) Comparison of RGC survival in the superior versus inferior retinal quadrants after pONT in the DA rat . (d) RGC density, (e) nearest neighbour distance and (f) regularity index in the superior (white) and inferior (black) retinal quadrants as outlined in Figure 1. RGC loss was significantly greater in the superior retina (paired two-tailed $T$-test) compared with the inferior retinal quadrant. (g) Further segmentation of RGC populations in the superior and inferior quadrants into a series of 15 non-overlapping concentric rings centred on the ONH suggest the greatest loss of RGC density occurs in the superior retinal quadrant versus the inferior quadrant over the course of the pONT model (red) when compared with naïve controls (black). These observations are reflected in the change in NND and RI. Data are presented as means \pm S.E.

in the central retina compared with the retinal periphery over the course of this model.

Significant RGC loss occurs in contralateral eyes in the OHT model Analysis of contralateral untreated eyes from $\mathrm{OHT}$ cohorts was next performed (Figure 5). A significant decline in RGC density (Figure 5a) was observed from 7 days post OHT induction $(P<0.001)$ (in an IOP related relationship), but to a lesser extent than eyes which were subject to elevated IOP (average RGC density loss 84 days post OHT induction $23 \pm 5 \%$ versus $14 \pm 6 \%$ for $\mathrm{OHT}$ and contralateral eyes, respectively, Table 1). Average NND increased from 7 days but only reached significance $(P<0.01)$ versus naïve controls 56 days post OHT inductions $(14.42 \pm 0.18$ versus $15.44 \pm 0.37, P<0.01$; Figure $5 \mathrm{~b}$ ). A similar trend was observed in $\mathrm{RI}(2.86 \pm 0.03$ versus $2.51 \pm 0.18, P<0.05$; Figure $5 \mathrm{c})$, suggesting preservation of RGC retinal mosaic. Further segmentation of retinal whole-mounts into 15 concentric rings (Figure $5 \mathrm{~d}$ ) reveals that loss of RGC density and NND occurs primarily in the central retinal and that despite significant loss of RGCs, RI remains largely unaffected, indicating preservation of the retinal mosaic.

Pattern of RGC loss reveals primary and secondary degenerative changes in $\mathrm{OHT}$ model comparable to human optical coherence tomography retinal nerve fibre layer segmentation

Clinically, optical coherence tomography retinal nerve fibre layer (RNFL) thickness changes are assessed by quadrant over time. ${ }^{43,44}$ We next investigated whether quadrant analysis could be used to define the rate of RGC density loss. Mean RGC density in each quadrant ring was plotted over time (Figures $6 a$ and b) and fit to exponential decay equations (equations (4)-(6)). Results were used to construct colour maps of percentage RGC density loss and half-life and to estimate the percentage of primary degeneration in each ring segment (Figure $6 c$ ). Figure $6 c$ illustrates that in the pONT model, the greatest percentage of RGC density loss occurred in the superior and central retinal segments (red regions) and least in the inferior segment and retinal periphery (blue sectors). This observation broadly correlated with half-life observations which were generally lower in the superior and central sectors $\left(t_{1 / 2} \sim 4\right.$ days, yellow sectors) versus the inferior periphery sectors $\left(t_{1 / 2}>10\right.$ days, green sectors). Interestingly the lowest half-life of RGC density decay was observed in the central inferior retina ( $t_{1 / 2} \sim 9$ days, orange sectors). By comparison, in the OHT model, the greatest percentage loss of RGC density was observed in the superior and inferior retinal sectors ( $30 \%$ RGC density loss, red), versus the nasal and temporal quadrants (20-0\%, white-blue sectors). Half-lives of RGC decay in this model were less in the superior retina than in other sectors, (average $t_{1 / 2}$ superior retina $=2.5$ versus $4.7,9.2$ and 7.4 days in the inferior, nasal and temporal retina, respectively). This pattern of RGC loss is in agreement with previous observations using axonal sections and optical coherence tomography in the same rodent model of OHT, where greater RNFL thinning was observed in the superiorinferior axis versus the nasal-temporal axis. ${ }^{25,42}$ RNFL thinning has been suggested as a risk factor for future visual field loss. ${ }^{45}$ The pattern of RGC loss observed also closely matches the bowtie-like pattern of RNFL loss previously reported in human glaucoma patients ${ }^{46}$ and is supported by recent clinical observations where significant thinning of the RNFL in the superior and inferior retina was observed in a population of glaucoma suspects. ${ }^{47}$ 

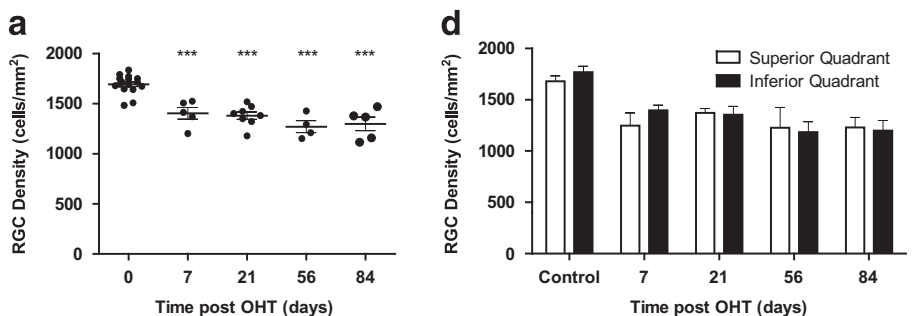

g
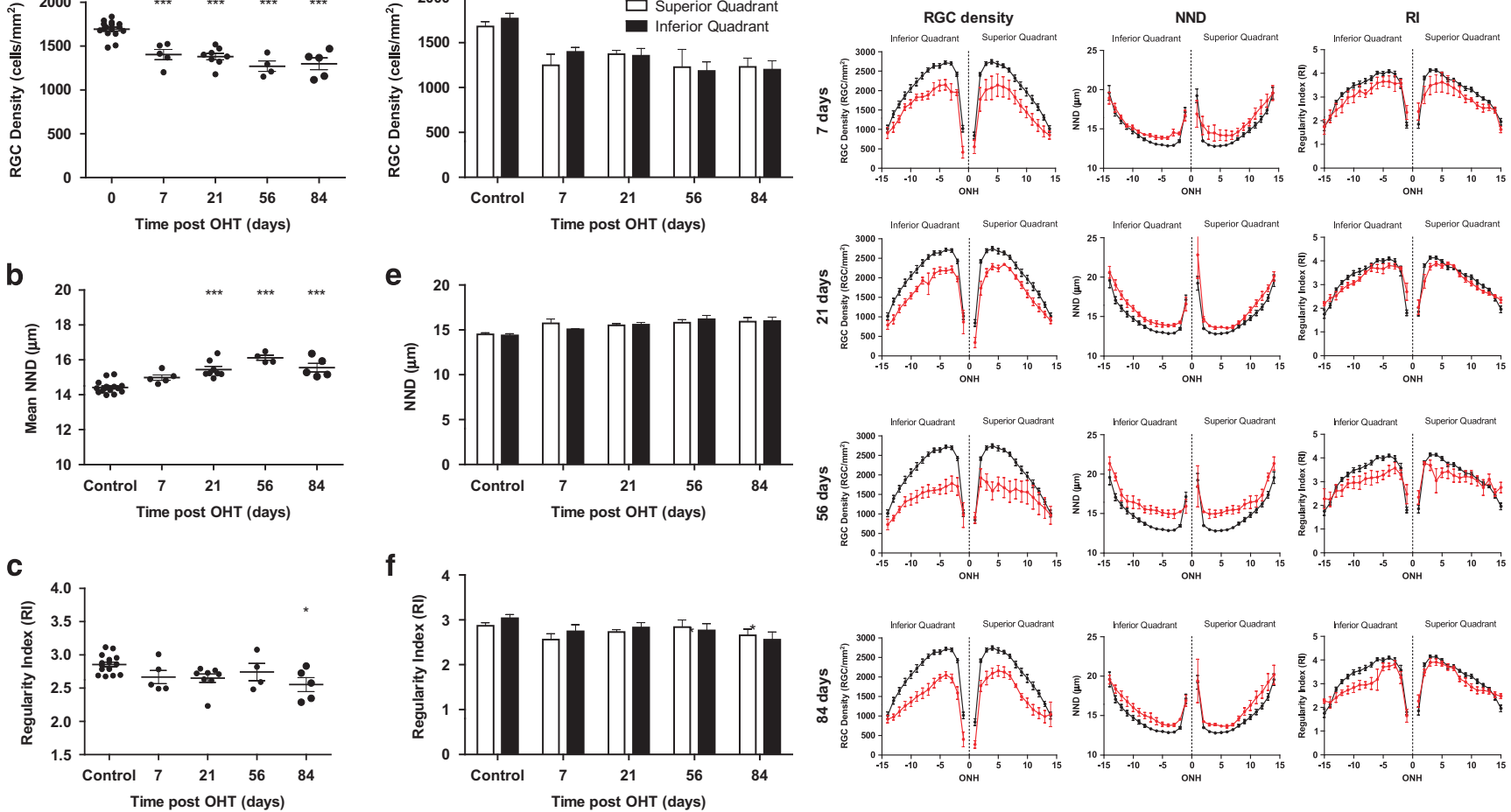

Figure 4. RGC survival after ocular hypertension (OHT) induction in the DA rat. Whole-retinal profiles of OHT induced loss of (a) RGC density, (b) nearest neighbour distance and (c) regularity index demonstrating significant reducing in RGC populations over the course of this model with only minor degradation in the regularity of the retinal mosaic (one-way ANOVA with Dunnets' post hoc test versus naïve controls). (d-f) Comparison of RGC survival in the superior versus inferior retinal quadrants after ocular hypertension (OHT) induction in the DA rat. Profile of OHT induced loss of (d) RGC density, (e) nearest neighbour distance and (f) regularity index in the superior (white) and inferior (black) retinal quadrants as outlined in Figure 1. No significant differences in RGC loss were detected between the superior and inferior retinal segments (paired two-tailed T-test). (g) Further segmentation of RGC populations in the superior and inferior quadrants into a series of 15 non-overlapping concentric rings centred on the ONH suggest that a similar extent of RGC density loss occurs in the superior and inferior retinal quadrants over the course of this model (red) when compared with naïve controls (black). These observations are reflected in the change in NND and RI. Data are presented as means \pm S.E.

Finally, to estimate the percentage of primary and secondary degeneration in each retinal sector, RGC density decay curves were fit to a two-phase exponential decay model, fixing rates of RGC loss at $t_{1 / 2}=1.7$ and 16.3 days for primary and secondary RGC degeneration as reported previously. ${ }^{48}$ Figure $6 \mathrm{c}$ illustrates that in the pONT model of retinal degeneration, a combination of primary and secondary degeneration was observed in the superior and central retina (65\% primary degeneration, green sectors) and the proportion of primary degeneration declined on approaching the superior retinal periphery ( $46 \%$ primary degeneration, yellow sectors). Surprisingly, the greatest occurrence of primary degeneration was observed in the central inferior retina, peaking at $84 \%$ in ring 4, while the lowest incidence of primary degeneration was observed in the interior, nasal and temporal retinal periphery (20-0\%, orange sectors).

\section{DISCUSSION}

This study describes a method for segmentation of Brn3a-labelled retinal whole-mounts to extract greater information regarding the spatial distribution and rate of RGC loss in the pONT and OHT models of retinal neurodegeneration than has previously been achieved (Figure 1). Automated analysis of retinal whole-mounts is preferable to manual counting of retinal sectors, as it minimises sampling error, unintentional bias and inter-operator variability. Using spatial and temporal analysis of RGC loss in these models, we reveal distinctive patterns of primary and secondary degeneration that correlate with previous observations and RNFL changes in clinical glaucoma. Finally, significant RGC loss was reported in the contralateral eyes of the OHT model, lending support to the hypothesis that systemic effects can exacerbate secondary RGC degeneration in clinical glaucoma. ${ }^{49}$

Using the algorithm described, naïve retina were found to possess a comparable number of RGCs and global RGC density as in previous studies using similar rat species. ${ }^{50,51}$ Over the course of the pONT model, RGC density loss was most pronounced in the superior retina in agreement with previous studies. $71,48,52$ However, in the present study, a significant reduction in RGC density was also observed in the inferior retinal segment as soon as 3 days post pONT induction, which is much earlier than the 28 days previously reported by Levkovitch-Verbin et al. (Figure 3). An explanation for this observation is that the Levkovitch-Verbin study utilised the retrograde Fluorogold labelling rather than Brn3a, which has previously been reported to provide an earlier indicator of RGC loss in axonal injury models. ${ }^{50}$ The observation that RGC numbers decline rapidly in all regions of the retina after pONT is in agreement with several recent studies, which report the rapid spread of oxidative and excitotoxic stress beyond the initial site of injury and mitochondrial changes within 24h of model induction. ${ }^{53-55}$ RGC density loss in both models was found to fit best to an exponential decay model, in common with previous studies of rodent ON crush and transection models. ${ }^{52,56}$

The algorithm uses concentric rings to divide retinal wholemounts into a series of 60 non-overlapping sectors, from which the regional spatial and temporal pattern of RGC loss can be evaluated. The advantage of this method of analysis, compared with using a series of concentric circles, is that changes in the central retina can be effectively differentiated from peripheral 


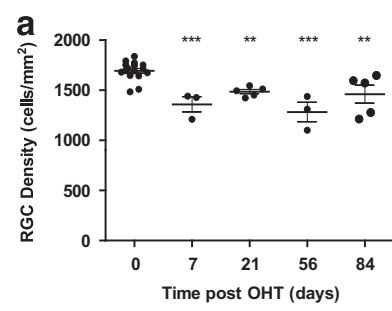

d
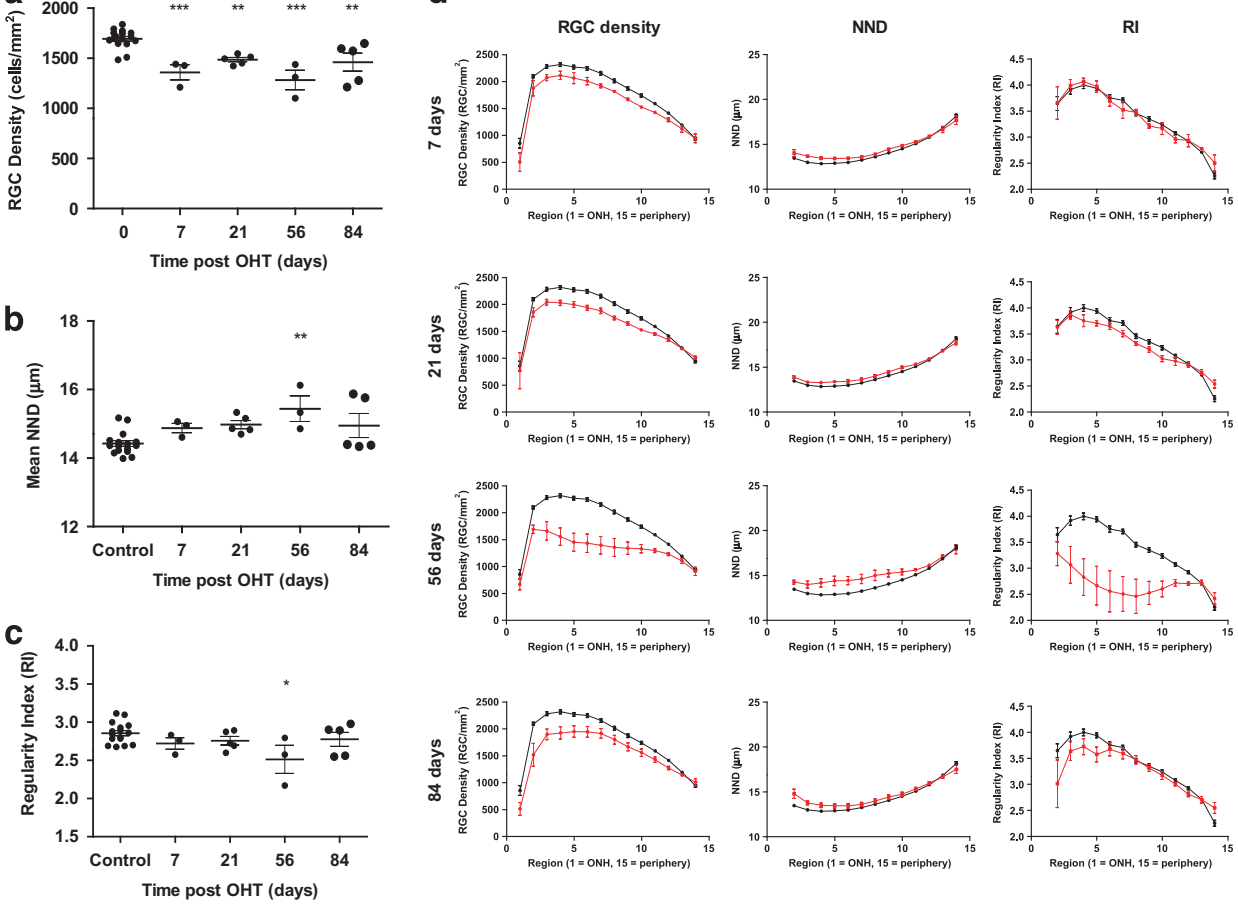

Figure 5. Retinal ganglion cell (RGC) survival after contralateral ocular hypertension (OHT) induction in the DA rat. Whole-retinal profile of (a) RGC density, (b) nearest neighbour distance (NND) and (c) regularity index demonstrating significant reducing in RGC populations when $\mathrm{OHT}$ was inducted in the opposite eye (Figure 4) over the course of this model with only minor degradation in the regularity of the retinal mosaic (one-way ANOVA with Dunnets' post hoc test versus naïve controls). (d) Segmentation of RGC populations into a series of 15 nonoverlapping concentric rings centred on the ONH suggest that greatest loss of RGC density occurs in the central versus the peripheral retina over the course of the OHT model (red) when compared with naïve controls (black). These observations are reflected in the change in NND and RI. Data are presented as means \pm S.E.

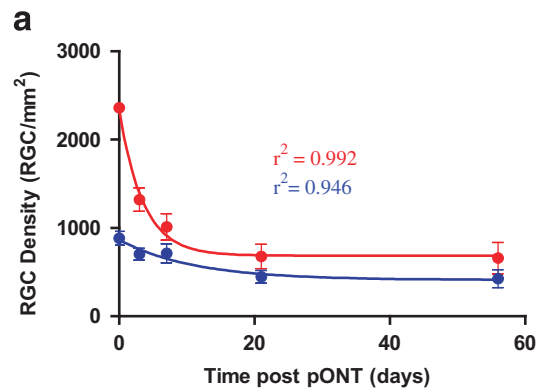

C $\%$ density loss
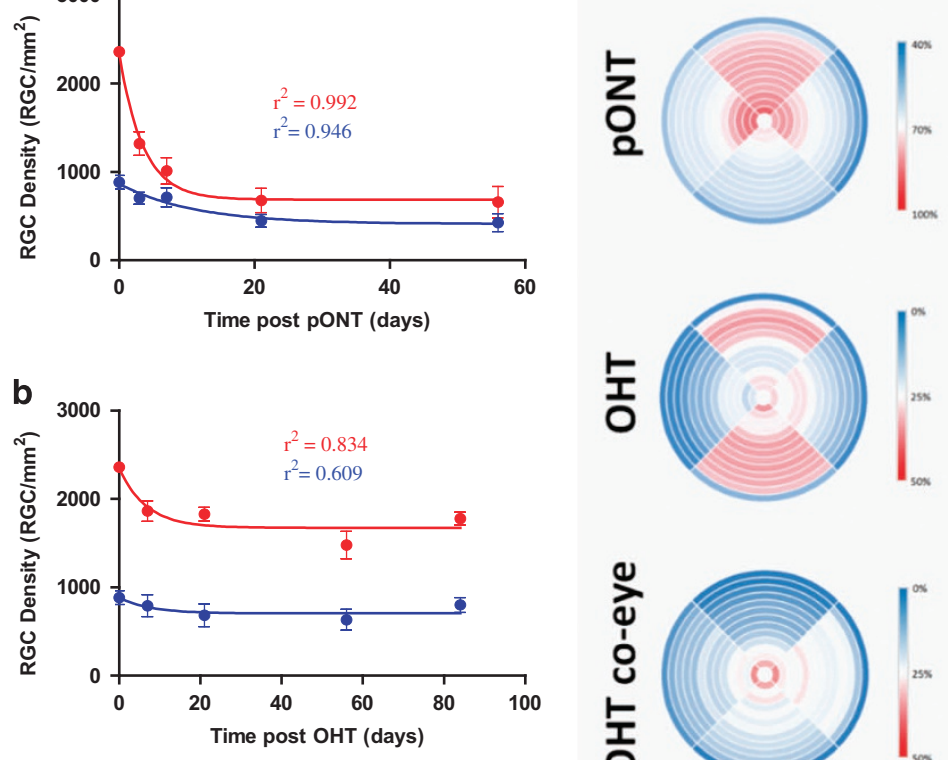

$\rightarrow-\operatorname{InfQ}$ Ring 4

$\rightarrow$ InfQ Ring 14

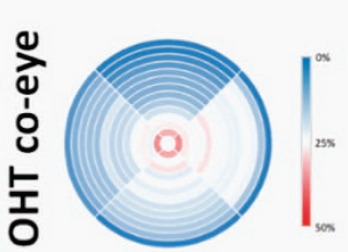

$t_{1 / 2}$
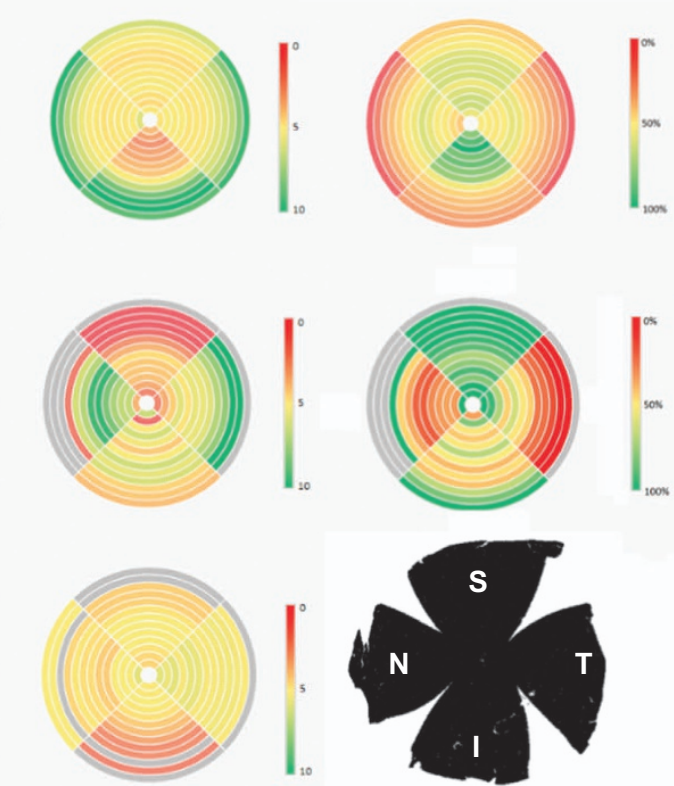

Figure 6. Summary of the rates of RGC loss in retinal segments of the pONT and OHT models. Example profiles of RGC density loss in the central (Ring 4, red) and peripheral (Ring 14, blue) inferior retina form the (a) pONT and (b) OHT models fit to a single-phase exponential decay model (equation (3)). (c) These data were used to construct colour maps summarising the percentage of RGC density loss over the course of each model, the half-life of RGC loss in each retinal sector on fitting to a single exponential decay model (equation (3)) and estimate the percentage of primary degeneration on fitting to a two-phase decay model (equations (4)-(6) as described in the text. 
retinal effects, an approach that has previously been used to eliminate point-pattern bias from Ripley's K-function. ${ }^{57}$

Analysis of regional RGC loss revealed that RGC density declined more substantially in the central retinal sectors than the peripheral retina in the pONT model (Figures 3 and 6) is in agreement with previous observations using axotomy models. ${ }^{29}$ By comparison, RGC loss in the OHT model was milder than that observed for the pONT model, reflecting the duration (3 weeks) and magnitude of IOP elevation of the OHT model, in agreement with previous studies. ${ }^{42}$ RGC loss in this model was concentrated in the central retina (visual streak). The observation of central retinal defects in both models of retinal degeneration is in broad agreement with previous studies using rodent models of $\mathrm{OHT}$ and axonal injury. ${ }^{29,58}$ This is of particular interest, as it is increasingly recognised that patients with glaucoma present with more complex retinal-wide visual defects than solely loss of peripheral vision, ${ }^{59}$ and coincides with a growing body of evidence suggesting that early clinical glaucomatous damage occurs in the macular, the site of greatest RGC density in humans. ${ }^{60}$

Secondary degeneration refers to the degeneration of RGCs in response to any consequence of the primary insult, such as oxidative stress or release of intracellular glutamates reserves, which can occur rapidly after onset of injury.,33,61 An advantage of the pONT model of RGC degeneration is that primary and secondary degeneration are considered spatially segregated, with approximately $75 \%$ of RGC loss in the superior retina but only $25 \%$ of RGC loss in the inferior retina reported to be a result of primary degeneration. ${ }^{19}$ In the present study, the half-life of RGCs in the pONT model was substantially less in superior and central retinal sectors versus the inferior, nasal and temporal retinal periphery, suggesting greater primary degeneration indeed occurs in these regions. A combination of primary and secondary degeneration was found to occur in both superior and inferior sectors of the pONT model in broad agreement with previous observations. ${ }^{19}$ However the observation that the central retina showed greater proportion of RGC loss than the peripheral retina in this model was unexpected and may be because the DA rat exhibit less ectopic axonal organisation than other species. ${ }^{31}$ By comparison, reductions in RGC density in the OHT model were mirrored by significant increases in mean NND. The change in distribution of normalised NNDs suggested a similar combination of clustered and diffuse RGC loss as observed in the pONT model, albeit to a lesser extent, reflecting the less severe insult. To the authors' knowledge, this study presents the first evidence to suggest primary and secondary degenerative processes in the rat OHT model.

Contralateral eyes are frequently used as untreated controls in animal models of glaucoma. This study reports that eyes contralateral to those subject to short-term IOP elevation were found to undergo significant RGC loss versus naïve eyes, despite exhibiting no significant change in IOP (Table 1 and Figure 5). RGC density loss in collateral eyes was less than that observed in eyes subject to increased IOP, but nevertheless significant compared with controls (Table 1). RGC density loss was most pronounced in the central retina (Figure 5). The pattern of RGC loss was different to that observed in OHT eyes, in that the pattern was less well defined with greatest loss in the nasal-temporal sectors (Figure 6). The surprising observation of RGC loss in contralateral eyes of rat glaucoma models is not without precedence. For example, unilateral optic nerve injury in the rat has previously been associated with bilateral activation of the pro-apoptotic transcription factor c-jun in RGCs, microglial activation and glia proliferation, ${ }^{62,63}$ and upregulation of stress response factor $\beta$ B2-crystallin; ${ }^{64}$ thought to play a role in neuroprotection and axonal regeneration. ${ }^{65}$ In murine models, contralateral upregulation of glial fibrillary acid protein, major histocompatibility complex-II (MHC-II) and the glial gap junction protein connexion 43 are reported, in addition to RGC loss. ${ }^{6-68}$ Several explanations have been proposed to describe the origin of the contralateral RGC degeneration observed in glaucoma models, including secretion of pro-inflammatory cytokines into the circulation, the propagation of degenerative signals from insulted to contralateral eyes via the visual centres of the brain or as a systemic autoimmune response. $^{69}$ The present study supports a growing body of evidence to suggest contralateral RGC loss in rodent models of retinal degeneration, and presents data to suggest that different patterns of RGC loss in OHT and contralateral eyes are observed in the well-established rat OHT model of glaucoma.

In conclusion, this article describes a novel algorithm to automatically extract information to a greater granularity than has previously been achieved from Brn3a-labelled retinal wholemounts. We show how this technique can be successfully used to investigate the extent and spatial segregation of primary and secondary degenerative processes in models of neurodegeneration. Furthermore, we demonstrate contralateral effects of OHT on RGC populations.

\section{MATERIALS AND METHODS}

\section{Animals}

Adult male Dark Agouti rats (150-200 g) were treated with procedures approved by the UK Home Office and in compliance with the ARVO Statement for the Use of Animals in Ophthalmic and Vision Research. All animals (80 rats, 100 eyes) were maintained in a $12 \mathrm{~h}$ light (140-260 lux)$12 \mathrm{~h}$ dark cycle, with food and water ad libitum.

\section{pONT model induction}

pONT was performed in the left eye of 35 DA rats, using an adaptation of a previously described technique., ${ }^{710}$ Briefly, under general anaesthesia, an incision was made in the superior conjunctiva, exposing the optic nerve sheath. The optic nerve was then exposed using a longitudinal slit in the dura mater to allow a $0.2 \mathrm{~mm}$ cut to be made in the dorsal optic nerve $2 \mathrm{~mm}$ behind the eye using an ophthalmic scalpel with a steel cutting guard. Damage to major ophthalmic blood vessels was avoided and verified at the end by ophthalmoscopy. Animals were killed 3, 7, 21 and 56 days post PONT surgery. A population of bilaterally untreated (naïve) retinas were used as controls.

\section{OHT model induction}

$\mathrm{OHT}$ was surgically induced in the left eye of 25 DA rats as described previously. ${ }^{42}$ IOP was elevated in the left eye of each animal by the injection of $0.05 \mathrm{ml}$ hypertonic saline solution $(1.80 \mathrm{M})$ into the two episcleral veins using a syringe pump $(0.05 \mathrm{ml} / \mathrm{min}$; UMP2; World Precision Instruments, Sarasota, FL, USA). A propylene ring, with a 1-mm gap cut out of its circumference, was placed around the equator to prevent injected saline outflow from other aqueous veins.

The IOP of both eyes in each rat was measured at regular intervals with a tonometer (TonoLab; Tiolat Oy, Heisinki, Finland) under inhalational anaesthesia ( $0.4 \%$ isoflurane in oxygen). For each animal, cumulative IOP exposure, defined as the integral of IOP elevation over time ( $\mathrm{mm} \mathrm{Hg} /$ day), was calculated from the area under the curve, as previously described. Animals were killed 7, 21, 56 and 84 days post-surgery. A population of bilaterally untreated (naïve) retinas were used as controls.

\section{Brn3a immunohistochemistry and confocal microscopy}

After animals were killed, both eyes were enucleated and fixed in $4 \%$ paraformaldehyde at $4{ }^{\circ} \mathrm{C}$ overnight before dissecting retinal wholemounts. Whole-mounts were stained for the RGC specific nuclear-localised transcription factor Brn3a using an anti-mouse mAb (1:500; Merk Millipore, Darmstadt, Germany) and examined under confocal microscopy (LSM 710; Carl Zeiss Microlmaging GmbH, Jena, Germany). Each retinal whole-mount was imaged as a tiled $z$-stack at $\times 10$ magnification, which was used to generate a single plane maximum projection of the RGC layer in each retina for subsequent analysis (Figure 1a). Each whole-mount image was manually orientated so that the superior retina was towards the top of the image using in vivo CSLO imaging of retinal vasculature as a reference. Retina image acquisition settings were kept constant for all retinas imaged, allowing comparison of Brn3a expression in each experimental group as previously described. ${ }^{50}$ 
Automated quantification of Brn3a-labelled RGCs in retinal whole-mounts

Quantification of Brn3a-labelled RGCs in retinal whole-mounts was achieved using an algorithm previously described. ${ }^{10}$ Briefly, a high-pass filter was first applied to the 8-bit Brn3a-labelled channel to remove background followed by application of a 130 intensity threshold. The ImageJ watershed algorithm was then used to separate touching particles and those within 7-21 $\mu \mathrm{m}$ size range were counted based on RGC sizes previously reported. ${ }^{34}$ Only particles with a circularity $>0.7$ were considered to be RGCs. Once identified, a region of interest was defined for each RGC (Figure 1c) from which RGC area, mean grey pixel intensity and centroid $(x, y)$ were recorded. The NND was determined for each RGC using an ImageJ macro developed by Yuxiong Mao (https://icme.hpc. msstate.edu/mediawiki/index.php/Nearest Neighbor_Distances_Calculatio n_with_ImageJ) from which RI was calculated as described previously (equation (1)), where $x$ is the mean nearest neighbour distance and $\sigma$ is the standard deviation of nearest neighbour distance. ${ }^{70}$

$$
\mathrm{RI}=\frac{x_{\mathrm{NND}}}{\sigma_{\mathrm{NND}}}
$$

Whole-mount retinal area determination and segmentation

The area of each retina was determined by manually applying a low intensity threshold (0-5) to each image to create a mask of retinal wholemount area (Figure 1b). Retinal area (black pixels) was measured using ImageJ as described previously. ${ }^{10}$ To determine the area of segmented retina, a series of 15 concentric rings of increasing radii $(0.3 \mathrm{~mm})$ centred on the $\mathrm{ONH}$ were applied to the whole-retinal mask and the areas of each subdomain were determined (Figure 1c). Rings were further subdivided into quadrants (Figure 1d) using the centre of the $\mathrm{ONH}(a, b)$ as the retinal centre in each case.

\section{Automated Brn3a-labelled RGC retinal segmentation}

Once identified, RGCs were further segmented into a series of 15 concentric rings of increasing radii $(0.6 \mathrm{~mm})$ centred on $(a, b)$. This was achieved by calculating the Euclidean distance of each RGC centroid relative to the $\mathrm{ONH}$ centre using equation $(2)$, where $(x, y)$ is the centroid of each RGC.

$$
d=\sqrt{(x-a)^{2}+(y-b)^{2}}
$$

For whole-ring retinal analysis the Euclidean distance $[D]$ of each RGC was used to gate cells into a series of 15 concentric rings $0.6 \mathrm{~mm}$ in radii centred on the $\mathrm{ONH}$. Rings were labelled 1 to 15 with ring 1 centred on the $\mathrm{ONH}$ and 15 on the retinal peripheral (Figure 1c). For quadrant analysis (Figures 1d and e), RGCs were gated into one of four quadrants: Temporal, Superior, Inferior and Nasal using Cartesian or polar coordinate based segmentation which gave identical results.

Mean RGC density was determined for each ring or by dividing the RGC count in each sector by the corresponding retinal area. Mean NND for each region was calculated by averaging NNDs for RGCs within each sector. In each case RI was calculated from mean NND and $\sigma_{\mathrm{NND}}$ of each sector, as described above. Colour mapped scatterplots were drawn for qualitative analysis using RGC centroid and NND values with Origin 2015 (OriginLabs, Northampton, MA, USA).

Determining the rate of RGC primary and secondary degeneration To determine half-life of RGC loss in both models, longitudinal profiles of RGC density in each retinal segment were fit to a one-phase exponential decay model with plateau (equation (3)) (Figure 6).

$$
\mathrm{y}=(\mathrm{YO}-\text { Plateau }) \times \exp (-K \times X)+\text { Plateau }
$$

To determine the percentage of primary degeneration in each retinal segment, aforementioned RGC density profiles were fit to a two-phase exponential decay model with plateau (equations (4)-(6)). Results were presented as colour maps (Figure $6 c$ ),

$$
\begin{gathered}
S_{\mathrm{F}}=(\mathrm{Y} 0-\text { Plateau }) \times P_{\mathrm{F}} \times 0.01 \\
S_{\mathrm{S}}=(Y 0-\text { Plateau }) \times\left(100-P_{\mathrm{F}}\right) \times 0.01 \\
y=\left(\text { Plateau }+S_{\mathrm{F}}\right) \times \exp \left(-K_{\mathrm{F}} \times X\right)+\left(\text { Plateau }+S_{\mathrm{S}}\right) \times \exp \left(-K_{\mathrm{S}} \times X\right)
\end{gathered}
$$

where $K_{\mathrm{F}}$ and $K_{\mathrm{s}}$ refer to the two rate constants fixed using the fast (primary degeneration) and slow (secondary degeneration) half-lives as described in the text, $S_{F}$ and $S_{S}$ refer to the span (distance between $Y_{0}$ and Plateau) accounted by the fast and slow components of the equation, and $P_{\mathrm{F}}$ is the percentage of primary degeneration.

\section{Statistical analysis}

All data were analysed with the Student's $t$-test or one-way ANOVA with Dunetts post hoc test versus control groups using GraphPad Prism 5 (GraphPad Software, Inc., La Jolla, CA, USA), unless described otherwise. The longitudinal profiles of RGC degeneration following PONT and OHT induction were fitted with a one-phase and a two-phase exponential decay model with plateau as described in the text. Data were presented as means \pm S.E. and $P<0.05$ was considered significant.

\section{ACKNOWLEDGEMENTS}

This work was supported by a MRC Confidence in concept award (REF: MC_PC_14118 v.2). We thank Dr Lies De Groef for useful comments in the preparation of this manuscript.

\section{COMPETING INTERESTS}

The authors declare no conflict of interest.

\section{REFERENCES}

1 LaPlaca MC, Simon CM, Prado GR, Cullen DK. CNS injury biomechanics and experimental models. Prog Brain Res 2007; 161: 13-26.

2 Yamaura I, Yone K, Nakahara S, Nagamine T, Baba H, Uchida K et al. Mechanism of destructive pathologic changes in the spinal cord under chronic mechanical compression. Spine (Phila Pa 1976) 2002; 27: 21-26

3 O'Dwyer L, Lamberton F, Bokde ALW, Ewers M, Faluyi YO, Tanner C et al. Using diffusion tensor imaging and mixed-effects models to investigate primary and secondary white matter degeneration in Alzheimer's disease and mild cognitive impairment. J Alzheimers Dis 2011; 26: 667-682.

$4 \mathrm{Kim}$ YS, Joh TH. Microglia, major player in the brain inflammation: their roles in the pathogenesis of Parkinson's disease. Exp Mol Med 2006; 38: 333-347.

5 Crowe MJ, Bresnahan JC, Shuman SL, Masters JN, Beattie MS. Apoptosis and delayed degeneration after spinal cord injury in rats and monkeys. Nat Med 1997; 3: 73-76.

6 Yin D, Yan X, Fan M, Hu Y, Men W, Sun L et al. Secondary degeneration detected by combining voxel-based morphometry and tract-based spatial statistics in subcortical strokes with different outcomes in hand function. Am J Neuroradiol 2013; 34: 1341-1347.

7 Levkovitch-Verbin H, Quigley HA, Martin KR, Zack DJ, Pease ME, Valenta DF. A model to study differences between primary and secondary degeneration of retinal ganglion cells in rats by partial optic nerve transection. IOVS 2003; 44: 3388-3393.

8 Zhang J, Zhang Y, Xing S, Liang Z, Zeng J. Secondary neurodegeneration in remote regions after focal cerebral infarction: a new target for stroke management? Stroke 2012; 43: 1700-1705.

9 Guimaraes JS, Freire MA, Lima RR, Souza-Rodrigues RD, Costa AM, dos Santos CD et al. Mechanisms of secondary degeneration in the central nervous system during acute neural disorders and white matter damage. Rev Neurol 2009; 48: 304-310.

10 Guo L, Davis B, Davis S, Nizari EM, Normando E, Shi H et al. Direct optic nerve sheath (DONS) application of Schwann cells prolongs retinal ganglion cell survival in vivo. Cell Death Dis 2014; 5: e1460.

11 Cordeiro MF, Guo L, Luong V, Harding G, Wang W, Jones HE et al. Real-time imaging of single nerve cell apoptosis in retinal neurodegeneration. Proc Natl Acad Sci 2004; 101: 13352-13356.

12 Kvam E, Nannenga BL, Wang MS, Jia Z, Sierks MR, Messer A. Conformational targeting of fibrillar polyglutamine proteins in live cells escalates aggregation and cytotoxicity. PLoS One 2009; 4: e5727.

13 Quigley HA. Glaucoma. Lancet 2011; 377: 1367-1377.

14 Quigley H, Nickells RW, Kerrigan L, Pease ME, Thibault DJ, Zack DJ. Retinal ganglion cell death in experimental glaucoma and after axotomy occurs by apoptosis. Invest Ophthalmol Vis Sci 1995; 36: 774-786.

15 Gupta N, Ang L-C, Noël de Tilly L, Bidaisee L, Yücel YH. Human glaucoma and neural degeneration in intracranial optic nerve, lateral geniculate nucleus, and visual cortex. Br J Ophthalmol 2006; 90: 674-678. 
16 Leske MC, Heijl A, Hyman L, Bengtsson B, Dong L, Yang Z. Predictors of long-term progression in the early manifest glaucoma trial. Ophthalmology 2007; 114: 1965-1972.

17 AGIS-Investigators. The advanced glaucoma intervention study (AGIS): 7. the relationship between control of intraocular pressure and visual field deterioration. Am J Ophthalmol 2010; 130: 429-440.

18 Anderson DR, Drance SM, Schulzer M. Comparison of glaucomatous progression between untreated patients with normal-tension glaucoma and patients with therapeutically reduced intraocular pressures. Am J Ophthalmol 1998; 126: 487-497.

19 Li H, Ruan Y, Ren C, Cui Q, So K. Mechanisms of secondary degeneration after partial optic nerve transection. Neural Regen Res 2014; 9: 565-574.

20 Galvao J, Davis BM, Cordeiro MF. In vivo imaging of retinal ganglion cell apoptosis. Curr Opin Pharm 2013; 13: 123-127.

21 Johnson TV, Tomarev SI. Rodent models of glaucoma. Brain Res Bull 2010; 81: 349-358.

22 Levkovitch-Verbin H, Quigley HA, Kerrigan-Baumrind LA, D'Anna SA, Kerrigan D, Pease ME. Optic nerve transection in monkeys may result in secondary degeneration of retinal ganglion cells. Invest Ophthalmol Vis Sci 2001; 42: 975-982.

23 Fitzgerald M, Bartlett CA, Evill L, Rodger J, Harvey AR, Dunlop SA. Secondary degeneration of the optic nerve following partial transection: the benefits of lomerizine. Exp Neurol 2009; 216: 219-230.

24 Agudo M, Pérez-Marín MC, Lönngren U, Sobrado P, Conesa A, Cánovas I et al. Time course profiling of the retinal transcriptome after optic nerve transection and optic nerve crush. Mol Vis 2008; 14: 1050-1063.

25 Morrison JC, Moore CG, Deppmeier LM, Gold BG, Meshul CK, Johnson EC. A rat model of chronic pressure-induced optic nerve damage. Exp Eye Res 1997; 64: $85-96$.

26 Guo L, Moss SE, Alexander RA, Ali RR, Fitzke FW, Cordeiro MF. Retinal ganglion cell apoptosis in glaucoma is related to intraocular pressure and IOP-induced effects on extracellular matrix. Invest Ophthalmol Vis Sci 2005; 46: 175-182.

27 Ji J, Chang P, Pennesi ME, Yang Z, Zhang J, Li D et al. Effects of elevated intraocular pressure on mouse retinal ganglion cells. Vis Res 2005; 45: 169-179.

28 Fortune B, Bui BV, Morrison JC, Johnson EC, Dong J, Cepurna WO et al. Selective ganglion cell functional loss in rats with experimental glaucoma. Invest Ophthalmol Vis Sci 2004; 45: 1854-1862.

29 Nadal-Nicolás FM, Jiménez-López M, Sobrado-Calvo P, Nieto-López L, CánovasMartínez I, Salinas-Navarro M et al. Brn3a as a marker of retinal ganglion cells: qualitative and quantitative time course studies in naive and optic nerve-injured retinas. Invest Ophthalmol Vis Sci 2009; 50: 3860-3868.

30 Kwong JMK, Quan A, Kyung H, Piri N, Caprioli J. Quantitative analysis of retinal ganglion cell survival with Rbpms immunolabeling in animal models of optic neuropathies. Invest Ophthalmol Vis Sci 2011; 52: 9694-9702.

31 Fitzgerald M, Payne SC, Bartlett CA, Evill L, Harvey AR, Dunlop SA. Secondary retinal ganglion cell death and the neuroprotective effects of the calcium channel blocker lomerizine. Invest Ophthalmol Vis Sci 2009; 50: 5456-5462.

32 Wang X, Li Y, He Y, Liang H-S, Liu E-Z. A novel animal model of partial optic nerve transection established using an optic nerve quantitative amputator. PLoS One 2012; 7: e44360.

33 Li H, Liang Y, Chiu K, Yuan Q, Lin B, Chang RC-C et al. Lycium barbarum (Wolfberry) reduces secondary degeneration and oxidative stress, and inhibits JNK pathway in retina after partial optic nerve transfection. PLoS One 2013; 8: e68881.

34 Danias J, Shen F, Goldblum D, Chen B, Ramos-Esteban J, Podos SM et al. Cytoarchitecture of the retinal ganglion cells in the rat. IOVS 2002; 43: 587-594.

35 Galindo-romero C, Jiménez-lópez M, García-ayuso D, Salinas-navarro M, Nadalnicolás F. Number and spatial distribution of intrinsically photosensitive retinal ganglion cells in the adult albino rat. Exp Eye Res 2013; 108: 84-93.

36 Valiente-Soriano FJ, Garcaa-Ayuso D, OrtÃn-MartÃnez A, Jimacnez-La-pez M, Galindo-Romero C, Villegas-Pacrez MP et al. Distribution of melanopsin positive neurons in pigmented and albino mice: evidence for melanopsin interneurons in the mouse retina. Front Neuroanat 2014; 8: 1-17.

37 Cook JE. Spatial properties of retinal mosaics: an empirical evaluation of some existing measures. Vis Neurosci 1996; 13: 15-30.

38 Wassle $\mathrm{H}$, Riemann HJ. The mosaic of nerve cells in the mammalian retina. Proc $R$ Soc B Biol Sci 1978; 200: 441-461.

39 Raven MA, Reese BE. Horizontal cell density and mosaic regularity in pigmented and albino mouse retina. J Comp Neurol 2002; 454: 168-176.

40 Lei Y, Garrahan N, Hermann B, Fautsch M, Johnson D, Hernandez M et al. Topography of neuron loss in the retinal ganglion cell layer in human glaucoma. Br J Ophthalmol 2009; 93: 1676-1679.

41 Filippopoulos T, Danias J, Chen B, Podos S, Mittag T. Topographic and morphologic analyses of retinal ganglion cell loss in old DBA / 2NNia mice. IOVS 2006; 47: 1968-1974.
42 Guo L, Normando EM, Nizari S, Lara D, Cordeiro MF. Tracking longitudinal retinal changes in experimental ocular hypertension using the cSLO and spectral domain-OCT. Invest Ophthalmol Vis Sci 2010; 51: 6504-6513.

43 Fortune B, Burgoyne CF, Cull G, Reynaud J, Wang L. Onset and progression of peripapillary retinal nerve fiber layer (RNFL) retardance changes occur earlier than RNFL thickness changes in experimental glaucoma. Invest Ophthalmol Vis Sci 2013; 54: 5653-5661.

44 Medeiros FA, Zangwill LM, Bowd C, Mansouri K, Weinreb RN. The structure and function relationship in glaucoma: implications for detection of progression and measurement of rates of change. Invest Ophthalmol Vis Sci 2012; 53: 6939-6946.

45 Quigley Ha, Katz J, Derick RJ, Gilbert D, Sommer A. An evaluation of optic disc and nerve fiber layer examinations in monitoring progression of early glaucoma damage. Ophthalmology 1992; 99: 19-28.

46 Quigley H, Dunkelberger GR, Green W. Chronic human glaucoma causing selectively greater loss of large optic nerve fibers. Ophthalmology 1988; 95: 357-363.

47 Khanal S, Thapa M, Racette L, Johnson R, Davey PG, Joshi MR et al. Retinal nerve fiber layer thickness in glaucomatous Nepalese eyes and its relation with visual field sensitivity. J Optom 2014; 7: 217-224.

48 Leung C, Lindsey J, Crowston J, Lijia C, Chiang S, Weinreb R. Longitudinal profile of retinal ganglion cell damage after optic nerve crush with blue-light confocal scanning laser ophthalmoscopy. IOVS 2008; 49: 4898-4902.

49 Sapienza A, Raveu A-L, Reboussin E, Roubeix C, Boucher C, Dégardin J et al. Bilateral neuroinflammatory processes in visual pathways induced by unilateral ocular hypertension in the rat. J Neuroinflammation 2016; 13: 44.

50 Nadal-Nicolás FM, Jiménez-López $M$, Salinas-Navarro $M$, Sobrado-Calvo $P$, Alburquerque-Béjar JJ, Vidal-Sanz $M$ et al. Whole number, distribution and coexpression of Brn3 transcription factors in retinal ganglion cells of adult albino and pigmented rats. PLoS One 2012; 7: e49830.

51 Salinas-Navarro $M$, Mayor-Torroglosa $S$, Jiménez-López $M$, Avilés-Trigueros $M$, Holmes $\mathrm{T}$, Lund $\mathrm{R}$ et al. A computerized analysis of the entire retinal ganglion cell population and its spatial distribution in adult rats. Vis Res 2009; 49: 115-126.

52 Chauhan BC, Stevens KT, Levesque JM, Nuschke AC, Sharpe GP, O'Leary N et al. Longitudinal in vivo imaging of retinal ganglion cells and retinal thickness changes following optic nerve injury in mice. PLoS One 2012; 7: e40352.

53 Fitzgerald M, Bartlett Ca, Evill L, Rodger J, Harvey AR, Dunlop SA. Secondary degeneration of the optic nerve following partial transection: the benefits of lomerizine. Exp Neurol 2009; 216: 219-230.

54 O'Hare Doig RL, Bartlett Ca, Maghzal GJ, Lam M, Archer M, Stocker R et al. Reactive species and oxidative stress in optic nerve vulnerable to secondary degeneration. Exp Neurol 2014; 261: 136-146.

55 Cummins N, Bartlett Ca, Archer M, Bartlett E, Hemmi JM, Harvey AR et al. Changes to mitochondrial ultrastructure in optic nerve vulnerable to secondary degeneration in vivo are limited by irradiation at $670 \mathrm{~nm}$. BMC Neurosci 2013; 14: 98.

56 Leung CK, Lindsey JD, Crowston JG, Lijia C, Chiang S, Weinreb RN. Longitudinal profile of retinal ganglion cell damage after optic nerve crush with blue-light confocal scanning laser ophthalmoscopy. Invest Ophthalmol Vis Sci 2008; 49: 4898-4902.

57 Wiegand T, Moloney KA. Rings, circles, and null-models for point pattern analysis in ecology. Oikos 2004; 104: 209-229.

58 Salinas-Navarro M, Alarcón-Martínez L, Valiente-Soriano FJ, Ortín-Martínez A, Jiménez-López $M$, Avilés-Trigueros $M$ et al. Functional and morphological effects of laser-induced ocular hypertension in retinas of adult albino Swiss mice. Mol Vis 2009; 15: 2578-2598.

59 Hu CX, Zangalli C, Hsieh M, Gupta L, Williams AL, Richman J et al. What do patients with glaucoma see? Visual symptoms reported by patients with glaucoma. Am J Med Sci 2014; 348: 403-409.

60 Hood DC, Raza AS, de Moraes CGV, Liebmann JM, Ritch R. Glaucomatous damage of the macula. Prog Retin Eye Res 2013; 32: 1-21.

61 Fitzgerald M, Bartlett CA, Harvey AR, Dunlop SA. Early events of secondary degeneration after partial optic nerve transection: an immunohistochemical study. $J$ Neurotrauma 2010; 452: 439-452.

62 Bodeutsch N, Siebert H, Dermon C, Thanos S. Unilateral injury to the adult rat optic nerve causes multiple cellular responses in the contralateral site. J Neurobiol 1999; 38: 116-128.

63 Panagis L, Thanos S, Fischer D, Dermon CR. Unilateral optic nerve crush induces bilateral retinal glial cell proliferation. Eur J Neurosci 2005; 21: 2305-2309.

64 Chiu K, Zhou Y, Yeung S-C, CK-M Lok, Chan OO-C, Chang RC-C et al. Up-regulation of crystallins is involved in the neuroprotective effect of wolfberry on survival of retinal ganglion cells in rat ocular hypertension model. J Cell Biochem 2010; 110: 311-320.

65 Thanos S, Böhm MRR, Meyer Zu Hörste M, Prokosch-Willing V, Hennig M, Bauer D et al. Role of crystallins in ocular neuroprotection and axonal regeneration. Prog Retin Eye Res 2014; 42: 145-161. 
66 Kuehn MH, Kim CY, Jiang B, Dumitrescu AV, Kwon YH. Disruption of the complement cascade delays retinal ganglion cell death following retinal ischemia-reperfusion. Exp Eye Res 2008; 87: 89-95.

67 Gallego BI, Salazar JJ, de Hoz R, Rojas B, Ramírez Al, Salinas-Navarro M et al. IOP induces upregulation of GFAP and MHC-II and microglia reactivity in mice retina contralateral to experimental glaucoma. J Neuroinflammation 2012; 9: 92.

68 Kerr NM, Johnson CS, Zhang J, Eady EK, Green CR, Danesh-Meyer HV. High pressureinduced retinal ischaemia reperfusion causes upregulation of gap junction protein connexin43 prior to retinal ganglion cell loss. Exp Neurol 2012; 234: 144-152.

69 Sambasivarao SV. Immune phenomena in glaucoma and conformation disorders: why is the second eye not involved? J Glaucoma 2014; 23: S59-S61.
70 Wässle H, Grünert U, Röhrenbeck J. Immunocytochemical staining of All-amacrine cells in the rat retina with antibodies against parvalbumin. J Comp Neurol 1993: 332: 407-420.

(i) This work is licensed under a Creative Commons Attribution 4.0 International License. The images or other third party material in this article are included in the article's Creative Commons license, unless indicated otherwise in the credit line; if the material is not included under the Creative Commons license, users will need to obtain permission from the license holder to reproduce the material. To view a copy of this license, visit http://creativecommons.org/licenses/ by/4.0/ 